\title{
Immunohistochemical study on survivin in sinonasal tumors and its relationship with the immunoexpression of Ki67 and Bcl-2
}

\author{
Olga Stasikowska-Kanicka1, Malgorzata Wagrowska-Danilewicz', Marian Danilewicz²
}

${ }^{1}$ Department of Nephropathology, Medical University of Lodz, Poland

${ }^{2}$ Department of Pathomorphology, Medical University of Lodz, Poland

\begin{abstract}
The immunoexpression of the inhibitor of apoptosis protein survivin has been shown to be a significant prognostic factor in various human cancers. Immunohistochemical method was used to examine the expression of survivin, Ki67 and Bcl-2 in 20 cases of sinonasal inverted papillomas (IPs), 12 cases of sinonasal squamous cell carcinoma (SNCs) and 19 cases of nasal chronic sinusitis as a control. Nuclear immunostaining for survivin was observed in 14 of $20(70 \%)$ cases of sinonasal IPs and 10 of $12(83.4 \%)$ cases of SNCs. Apart from nuclear, also weak cytoplasmic immunoexpression of survivin was detected in 2 of 20 cases (10\%) of sinonasal IP and moderate intense staining in 9 of 12 cases $(75 \%)$ of SNC. There was no immunostaining for survivin in 19 control cases. The immunoexpression of survivin, Ki67 and Bcl-2 was significantly higher in SNCs than in sinonasal IPs and control group. Moreover, nuclear survivin and Ki67 antigen immunoexpression were significantly higher in sinonasal IPs group as compared to control group. There were statistically significant positive correlations between nuclear (but not cytoplasmic) immunoexpression of survivin and Ki67 antigen, as well as Bcl-2 oncoprotein in both tested tumors. In conclusion, our findings suggest that survivin, Ki67 and Bcl-2 may be involved in sinonasal tumorigenesis. (Folia Histochemica et Cytobiologica 2013, Vol. 51, No. 3, 225-231)
\end{abstract}

Key words: survivin, inverted papilloma, sinonasal cancer, Ki67, Bcl-2

\section{Introduction}

Cancers arising in the sinonasal cavity and surrounding tissues are extremely rare. It has been estimated that the incidence of sinonasal cancers is approximately $1 / 500,000$ to $1 / 1000,000$. These epithelial tumors occur most commonly in Caucasian race, in the fifth and sixth decades of life [1-4]. In comparison to other head and neck cancers, no predominant risk factors were described. There are some rare occupational and industrial exposures which may account for the development of these cancers. These include exposure to fumes, dusts from wood and leather and exposure to cadmium, nickel or chromium dusts and other rare

Correspondence address: O. Stasikowska-Kanicka,

Department of Nephropathology, Medical University of Lodz, Czechoslowacka St. 8/10, 92-216 Lodz, Poland

tel.|fax: +48 426790191

e-mail: olgast@op.pl minerals. As a consequence of industrial exposure, these malignancies appear to occur more commonly in males than females $(2: 1)$.

Sinonasal inverted papilloma (IP) is a benign epithelial neoplasm that arises from the outlining Schneiderian respiratory membrane. It is a rare sinonasal tumor accounting for only $0.5-4 \%$ of all nasal tumors. IPs generate considerable interest, because they have features of local invasiveness, tendency for recurrence and malignant transformation. The incidence of malignant transformation of IPs ranges from 2 to $27 \%$. It was reported that nearly $10 \%$ of IPs are associated with squamous cell carcinoma [5, 6]. However, the nature and pathogenesis of IPs as premalignant lesions are still debated.

Many studies have shown that the occurrence and development of malignant tumor are closely related to the overexpression of oncogenes and apoptosis inhibitory factors. Regulation of apoptosis is finely balanced by signaling pathways including apoptosis -promoting factors such as p53, Bax and caspases, and 
antiapoptotic factors such as Bcl-2 and MDM2 [7, 8]. A group of apoptosis inhibitor molecules, called inhibitor of apoptosis proteins (IAP), constitutes a family of evolutionarily conserved apoptosis suppressors; one member of the IAP family is survivin [9].

Survivin is a $16.5 \mathrm{kDa}$ protein, also called baculoviral inhibitor of apoptosis repeat-containing 5 (BIRC5). It contains only one baculovirus IAP repeat and lacks a carboxyl-terminal RING finger, which makes survivin different from other IAP proteins [9]. Survivin is strongly expressed during embryonic and fetal development. It is expressed in human fetal lung, heart, kidney, liver and gastrointestinal tract, and may contribute to tissue homeostasis and differentiation [10]. Survivin is rarely expressed in normal adult tissues except for the thymus, placenta and CD34+ stem cells [11]. The levels of survivin in normal adult cells are low in resting endothelial cells and could be up-regulated on activation to proliferation [9, 12]. Molecular mechanisms of the regulation of survivin expression in cancer are not clearly understood, but disruption of the survivin induction pathway has resulted in increased apoptosis and inhibition of tumor growth [12]. Numerous data demonstrate that survivin is highly expressed by the most common human neoplasms, including cancers of the lung, pancreas, stomach, colon, malignant melanoma, neuroblastoma, genito-urinary, hepatocellular and breast cancers, and soft tissue sarcomas $[13,14]$. Survivin is expressed in human cancer cells at a frequency of 34-100\% [15-17].

Survivin exists in two subcellular compartments - cytoplasmic and nuclear and in three splice variants: wild -type survivin, survivin-2B and survivin delta Ex3. Wild -type and survivin-2B variants are more often found in the cytoplasm, whereas the delta Ex 3 is more frequent in the nucleus [18]. Survivin plays a pro-mitotic and anti-apoptotic role $[19,20]$. Survivin is able to inhibit factors favorable to apoptosis for example, caspases. Survivin can also partially inhibit the cell death induced by Fas and Bax [15, 21]. Importantly, it is recognized that survivin not only inhibits apoptosis, but also, as a component of the chromosomal passenger complex, favors cancer cell proliferation [22-24]. During mitosis, survivin binds to and stabilizes mitotic spindles [25]. In the absence of survivin, cycling cells undergo mitotic collapse and caspase 9-mediated apoptosis [26, 27]. Upon the completion of mitosis, survivin is efficiently removed from the cell, so that cycling cells harvested in G1 no longer show survivin expression [28].

Little is known about the distribution of survivin in sinonasal lesions and how it correlates with other markers of tumorigenesis. Therefore, the objectives of this study were to evaluate the immunoexpression of survivin, Ki67 and Bcl-2 in cases with sinonasal inverted papillomas and sinonasal carcinomas. Another purpose was to find whether the immunoexpression of survivin could correlate with the immunoexpression of Ki67 and Bcl-2.

\section{Material and methods}

Patients. Twenty cases of sinonasal inverted papillomas, twelve cases of sinonasal squamous cell carcinomas (GII grade) and nineteen cases of chronic sinusitis as a control were retrieved from archival material (Chair of Pathomorphology, Medical University of Lodz, Poland). Tissue sections taken from postoperative material were diagnosed using a standard hematoxylin and eosin staining and the histological diagnoses were established according to the current standards [29]. A representative block of formalin fixed paraffin-embedded tissue from each case was selected and used for the study. The main criteria for patient's selection were histopathological similarities within the group and the same anatomical localization of lesions. The age range for sinonasal inverted papillomas was from 29 to 77 years $(55.8 \pm 12.82$, mean and SD), for sinonasal cancer was from 47 to 71 years $(61.1 \pm 9.94)$ and for chronic sinusitis was from 20 to $75(44.8 \pm 17.65)$ years.

Immunohistochemistry. $5 \mu \mathrm{m}$ sections were cut from blocks of formalin fixed paraffin-embedded tissue and mounted on microscope slides (SuperFrost Plus, Gerhord Menzel GmbH, Braunschweig, Germany), deparaffinized, rehydrated, then treated in a microwave oven in a solution of TRS (Target Retrieval Solution, Dako, Glostrup, Denmark) for 30 minutes $(2 \times 6$ minutes $360 \mathrm{~W}, 2 \times 5180 \mathrm{~W}, 2 \times 4$ minutes $90 \mathrm{~W})$ and transferred to distilled water. The endogenous peroxidase activity was blocked by incubation in $0.3 \%$ hydrogen peroxide in distilled water for 30 minutes, and then sections were rinsed with Tris-buffered saline (TBS, Dako) and incubated overnight with monoclonal anti-human survivin antibody (dilution 1:300, Abcam, Cambridge, UK), 30 minutes with mouse monoclonal anti-human antibodies: Ki67 (dilution 1:100, Dako) and Bcl-2 (dilution 1:50, Dako). Immunoreactive proteins were visualized using EnVision-HRP kit (Dako) according to the manufacturer's protocol. Visualization was performed by incubating the sections in a solution of 3,3'-diaminobenzidine (Dako). After washing, the sections were counter-stained with hematoxylin and coverslipped. For each antibody and for each sample negative controls were processed. Negative controls were carried out by incubation in the absence of the primary antibody and always yielded negative results.

The immunohistochemical expression of cytoplasmic survivin was evaluated semiquantitatively. Two independent observers scored immunolabeled sections using a scale ranging from 0 to $3(0$ - reaction not detectable, 1 - weak, 2 - moderate, 3 - intense reaction) in 7-10 high power fields. The mean grade was calculated by averaging grades assigned by the two observers and the mean approximated to the nearest unity. 
Morphometry. The immunohistochemical reactions for nuclear survivin, Ki67 and Bcl-2 were analyzed quantitatively using computer image analysis system consisting of a PC computer equipped with a Pentagram graphic tablet, Indeo Fast card (frame grabber, true-color, real-time), produced by Indeo (Taipei, Taiwan), and color TV camera Panasonic (Tokyo, Japan) coupled with Jenaval Carl Zeiss microscope (Carl Zeiss, Jena, Germany). This system was programmed (MultiScan 8.08 software, produced by Computer Scanning Systems, Warsaw, Poland) to calculate the number of objects (semiautomatic function).

The percentage of surviving positive (nuclear), Ki67 positive cells and Bcl-2 positive epithelial cells were estimated by counting 100 cells in five monitor fields $\left(0.029 \mathrm{~mm}^{2}\right.$ each $)$, marking immunopositive cells (semiautomatic function), so that in each case 500 cells were analyzed.

Statistical methods. The differences between groups were tested using unpaired Student's $t$-test preceded by evaluation of normality and Levene's test. The Mann-Whitney $U$-test was used for comparison of means between groups. Correlation coefficients were calculated using Spearman's method. Results were considered statistically significant if $\mathrm{p}<0.05$.

\section{Results}

The immunoexpression of survivin in epithelial tumor cells in patients with sinonasal IP and SNC was predominantly nuclear, although cytoplasmic expression was also noted. Nuclear immunoexpression of survivin was detected in 14 of 20 cases (70\%) with sinonasal IPs (Figure 1) and in 10 of 12 cases $(83,4 \%)$ of SNCs (Figure 2). Weak cytoplasmic immunoexpression of survivin was detected in 2 of 20 cases $(10 \%)$ with sinonasal IPs and moderate staining in 9 of 12 cases $(75 \%)$ of SNCs (Figure 3). In control patients there was no nuclear and no cytoplasmic immunostaining for survivin visible.

The results of morphometric analyses of the immunoexpression of survivin, Ki67 antigen and Bcl-2 oncoprotein in patients with sinonasal IP, SNC and in control cases are shown in Table 1 . The percen-

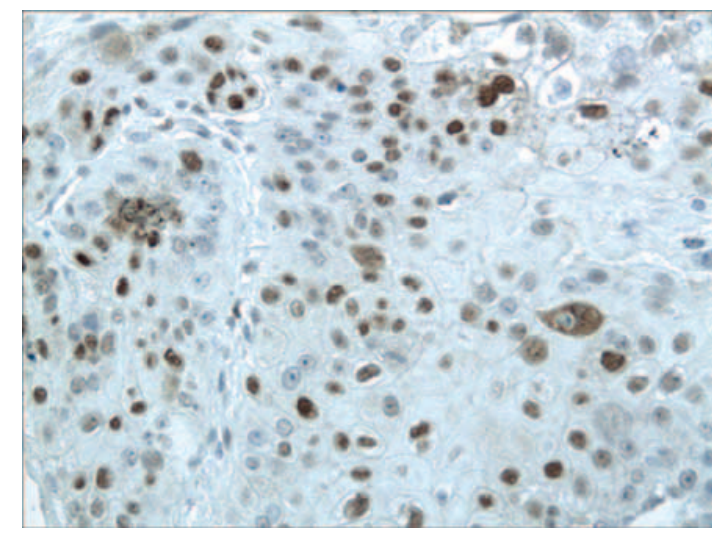

Figure 1. Nuclear and cytoplasmic immunoexpression of survivin in sinonasal inverted papilloma. Total magnification $\times 200$

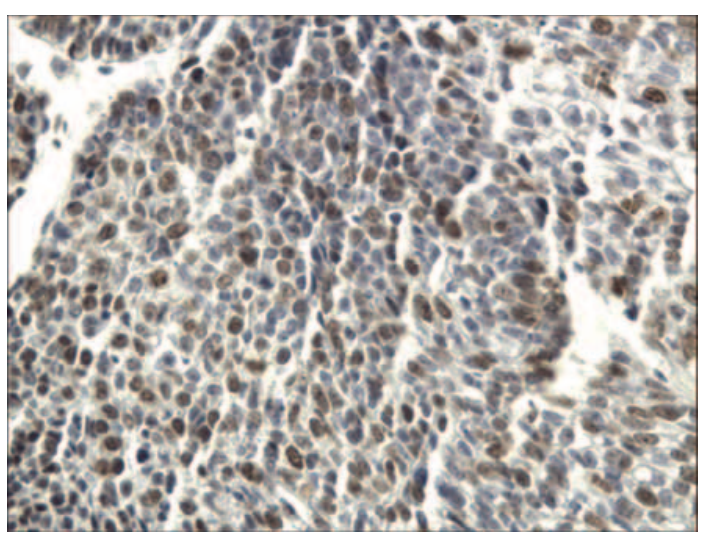

Figure 2. Nuclear immunoexpression of survivin in sinonasal squamous cell carcinoma. Immunohistochemistry. Total magnification $\times 200$

tages of epithelial tumor cells with nuclear survivin immunoexpression and the mean score of cytoplasmic immunoexpression of survivin in the SNC group were significantly increased as compared to sinonasal IP and control cases; in the latter the immunoexpression of survivin was entirely negative. The immunoexpresssion

Table 1. The immunoexpression of survivin, Ki67 antigen, and Bcl-2 oncoprotein in patients with sinonasal IP, sinonasal carcinoma (SNC) and controls

\begin{tabular}{|l|c|c|c|c|}
\hline Groups & Nuclear survivin (\%) & Cytoplasmic survivin (mean score) & Ki67 (\%) & Bcl-2 (\%) \\
\hline IP $(\mathrm{n}=20)$ & $1.08 \pm 0.81$ & $0.13 \pm 0.11$ & $17.62 \pm 6.27$ & $1.76 \pm 0.90$ \\
\hline SNC $(\mathrm{n}=12)$ & $3.75 \pm 2.01$ & $1.3 \pm 0.9$ & $45.24 \pm 9.97$ & $4.18 \pm 1.15$ \\
\hline Control $(\mathrm{n}=19)$ & $0.00 \pm 0.00$ & $0.00 \pm 0.00$ & $2.85 \pm 1.57$ & $1.23 \pm 1.03$ \\
\hline IP vs. SNC & $\mathrm{p}<0.001$ & $\mathrm{p}<0.001$ & $\mathrm{p}<0.001$ & $\mathrm{p}<0.001$ \\
IP vs. control & $\mathrm{p}<0.001$ & $\mathrm{p}=0.56(\mathrm{NS})$ & $\mathrm{p}<0.001$ & $\mathrm{p}=0.09$ (NS) \\
SNC vs. control & $\mathrm{p}<0.001$ & $\mathrm{p}<0.001$ & $\mathrm{p}<0.001$ & \\
\hline
\end{tabular}

NS - not significant 


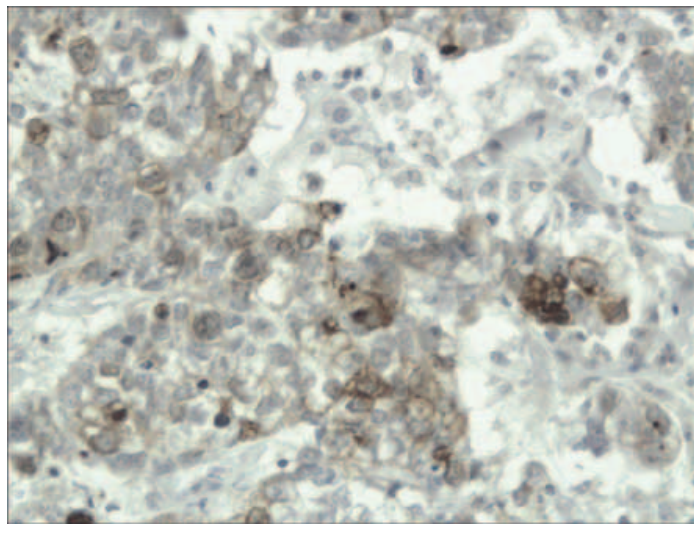

Figure 3. Cytoplasmic immunoexpression of survivin in sinonasal squamous cell carcinoma. Total magnification $\times 200$

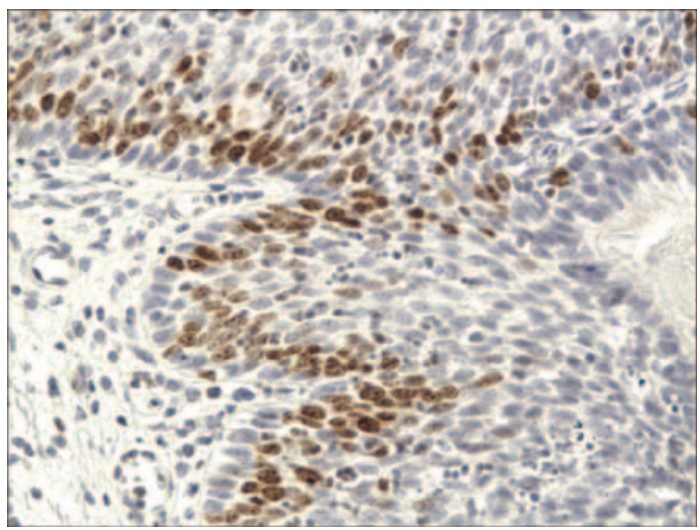

Figure 4. Immunoexpression of Ki67 in sinonasal inverted papilloma. Total magnification $\times 200$

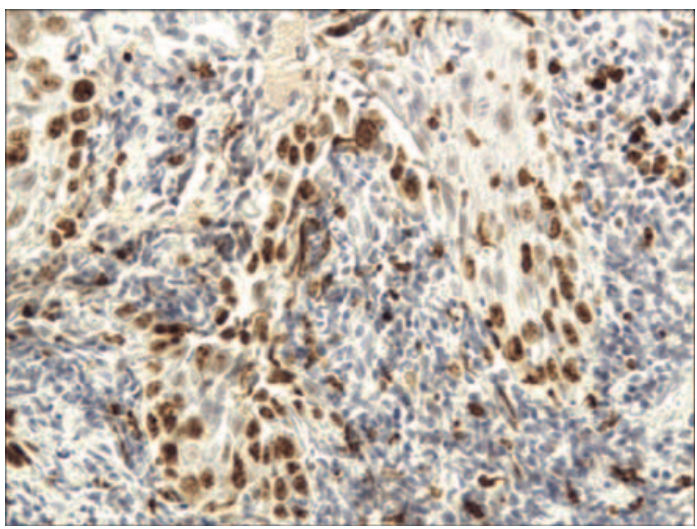

Figure 5. Immunoexpression of Ki67 in sinonasal squamous cell carcinoma. Total magnification $\times 200$

of survivin was also higher in sinonasal IPs group as compared to control group, but only nuclear survivin immunoexpression was significantly higher. The percentages

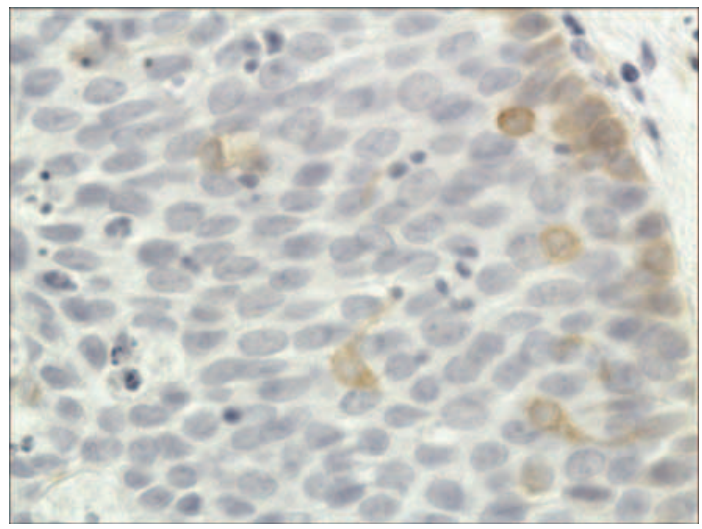

Figure 6. Immunoexpression of $\mathrm{Bcl}-2$ oncoprotein in sinonasal inverted papilloma. Total magnification $\times 400$

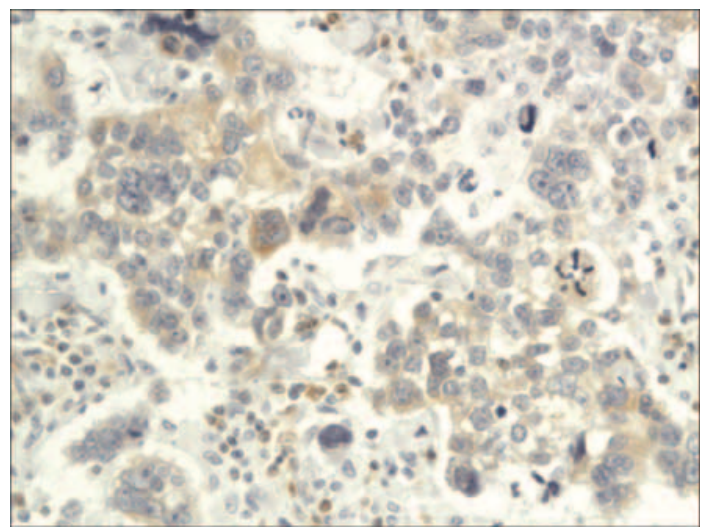

Figure 7. Immunoexpression of $\mathrm{Bcl}-2$ oncoprotein in sinonasal squamous cell carcinoma. Total magnification $\times 200$

of epithelial tumor cells with Ki67 immunoreactivity (Figures 4, 5) and Bcl-2 positive immunostaining (Figures 6,7$)$ in the SNC group were significantly increased as compared to both sinonasal IPs and control cases. The percentages of Ki67 positive epithelial tumor cells were also significantly higher in sinonasal IPs group as compared with control group. No statistically significant difference in Bcl-2 immunoexpression was noted between sinonasal IPs and control cases.

The correlations between the immunoexpression of survivin and Ki67 antigen and between survivin and Bcl-2 oncoprotein in patients with sinonasal IP and SNC are presented in Table 2. In the SNC and sinonasal IP groups there were statistically significant positive correlations between nuclear immunoexpression of survivin and Ki67 immunoreactivity, as well as Bcl-2 oncoprotein positivity, whereas in the SNC group the correlations between cytoplasmic immunoexpression of survivin and these parameters were not significant. 
Table 2. The correlations between immunoexpression of survivin and Ki67 antigen and Bcl-2 oncoprotein in patients with sinonasal inverted papilloma (IP) and sinonasal carcinoma (SNC)

\begin{tabular}{|l|c|c|}
\hline Correlation between & IP $(\mathbf{n}=\mathbf{2 0})$ & SNC $(\mathbf{n}=\mathbf{1 2})$ \\
\hline Nuclear survivin vs. Ki-67 & $\mathrm{r}=0.57$, & $\mathrm{r}=0.63$, \\
& $\mathrm{p}<0.009$ & $\mathrm{p}<0.03$ \\
\hline Nuclear survivin vs. Bcl-2 & $\mathrm{r}=0.84$, & $\mathrm{r}=0.72$, \\
& $\mathrm{p}<0.001$ & $\mathrm{p}<0.009$ \\
\hline Cytoplasmic survivin vs. Ki-67 & - & $\mathrm{r}=0.35$, \\
& & $\mathrm{p}=0.26(\mathrm{NS})$ \\
\hline Cytoplasmic survivin vs. Bcl-2 & - & $\mathrm{r}=0.27$, \\
& & $\mathrm{p}=0.39(\mathrm{NS})$ \\
\hline
\end{tabular}

NS — not significant

\section{Discussion}

Little is known about the biologic and prognostic significance of the survivin expression in sinonasal lesions. Moreover, data concerning correlation of survivin with Ki67 and Bcl-2 proteins in IPs and SNCs are notably scanty. Among many publications relevant to survivin localization in nuclei and cytoplasm in various cancers, part of them showed that the nuclear expression of survivin is an unfavorable prognostic marker, whereas the other proposed an opposing notion [30]. Our study showed significantly increased both nuclear and cytoplasmic immunoexpression of survivin in SNC patients as compared to sinonasal IPs and the control cases. Moreover, nuclear survivin immunoexpression was significantly increased in sinonasal IPs in comparison with the control group, in which the immunoexpression was entirely negative. Similarly to our results, Liang et al. [31], demonstrated survivin immunoexpression in $80 \%$ sinonasal squamous cell carcinoma and $73,3 \%$ of sinonasal IP and showed that survivin expression was significantly higher in squamous cell cancers and sinonasal IP than in control groups. Liang et al. [31] suggested that survivin may play an important role in the pathway of progression of sinonasal IP to SNC. In the study of cancer and precancerous lesions of oral cavity, it was shown that survivin was expressed in $33 \%$ of oral precancerous lesions and $94 \%$ of oral cancers [32]. The authors believed that strong nuclear and cytoplasmic immunoexpression of survivin is an early event during oral carcionogenesis [32]. Grabowski et al. [33] showed nuclear expression of survivin in $80 \%$ of cases of esophageal squamous cell carcinoma. Moreover, the survival of patients with nuclear survivin expression was significantly lower than that of patients without nuclear immunoexpression of survivin. The cytoplasmic staining for survivin had no prognostic significance.
In the present study, in all control cases of nasal chronic sinusitis the staining for survivin was totally negative (no nuclear and no cytoplasmic immunoreactivity). Other investigators also reported differential immunoexpression of survivin in cancer and normal tissues. For instance, survivin was expressed in $48 \%$ of sinonasal cancers, but no staining was present in normal tissue adjacent to the tumor [34]. Wang et al. [35] found survivin immunoexpression in laryngeal squamous cell carcinoma, but also did not reveal immunoexpression in normal laryngeal mucosa.

Literature data suggests higher expression of survivin (nuclear or cytoplasmic or both), as an apoptotic marker which correlates significantly with tumor grade, stage, and patient outcome including recurrence rate, and disease-free survival rate [12, 36-38]. Current reports in these research areas are inconsistent and propose opposing conclusions regarding the significance and prognostic value of nuclear and cytoplasmic immunoexpression of survivin [30]. Full understanding of whether the opposite effects seen with nuclear $v s$. cytoplasmic survivin are due to the subcellular localization or the differential functions of the splice variants remains to be elucidated. Possible explanation for part of these discordant results are histopathological differences between studied tumors, the low number of studied cases and methodological issues. We speculate, that differences concerning survivin immunoexpression described in our study may indicate that mechanisms responsible for the regulation of apoptosis in various tumors are different and not fully explored.

In this study, we have also observed that $\mathrm{Bcl}-2$ immunoexpression was statistically significantly increased in SNCs in comparison with sinonasal IPs and control group. Similar to our results, Liang et al. [31] showed significantly higher immunoexpression of Bcl-2 in cancers than in normal tissue. Katori et al. [39] demonstrated increased immunoexpression of Bcl-2 in sinonasal IPs with severe dysplasia and invasive cancer compared with control but also with sinonasal IP containing mild dysplasia. Our study did not reveal statistically significant differences of Bcl-2 immunoexpression between sinonasal IPs and control group. Surprising results were provided by Fan et al. [40] who found no differences regarding immunoexpression of Bcl-2 among SNC, sinonasal IP and sinonasal IP with dysplasia. In our study the high, nuclear survivin immunoexpression in SNC and sinonasal IP tissues was correlated with high immunoexpression of Bcl-2 oncoprotein. These results seem to be consistent with other studies $[31,35,37$, 41]. In contrary to above-mentioned results, Sun et al. [42] did not reveal correlation between survivin and 
Bcl-2 immunoexpression in 40 cases of laryngeal and hypopharyngeal cancers.

The Ki67 protein is a cellular marker for proliferation, present during all active phases of the cell cycle. In our study, the immunoexpression of Ki67 antigen was higher in SNC patients as compared to sinonasal IP and control group. This parameter was also significantly higher in sinonasal IP group in comparison with controls. Similarly to our study, other authors also demonstrated higher immunoexpression of Ki67 in squamous cell carcinoma and nasal IPs with dysplasia, suggesting that a high proliferative rate is a characteristic of IP-associated malignant diseases [43-47]. Kawasaki et al. [47] postulated survivin as the strongest apoptosis inhibitory factor, involved in the regulation of cellular proliferation in colon cancer. In our study, higher nuclear (but not cytoplasmic) survivin immunoexpression was correlated with higher Ki67 immunoexpression in both SNC and sinonasal IP tissues. To our knowledge, the data concerning relationship between these parameters in sinonasal lesions are scanty. In model cancer cells, expression of the survivin gene was shown to occur exclusively in the $\mathrm{G} 2 / \mathrm{M}$ phase in a strict cell cycle-regulated manner [48], thus potentially explaining a preferential expression of survivin in poorly differentiated and metastatic squamous cell cancers, likely to exhibit high proliferative potential. At a cellular level, survivin is localized to mitotic spindle microtubules of dividing cells [48], in a reaction required to preserve apoptosis inhibition. In this context, correlations described in our study may state logical consequence of the molecular events. Based on our results and literature data, we postulate that the nuclear survivin immunoexpression can be involved in promoting cell proliferation in sinonasal tumors, whereas the cytoplasmic survivin immunoexpression may be associated with clinicopathological parameters (not studied in this work).

In conclusion, our findings may suggest that relationship between survivin, Ki67 and Bcl-2 could potentially contribute to tumorigenesis in the sinonasal region. Profound analysis of molecular mechanism of action and subcellular localization of survivin and/or its presumptive variants may clarify the function of survivin in the regulation of cell viability and cell divisions. However, further studies are needed to better understand the molecular basis and the role of survivin in sinonasal tumorigenesis.

\section{Acknowledgment}

This study was supported by Medical University of Lodz, grant 502-03/6-038-01/502-64-022.
1. Caplan LS, Hall I, Levine RS et al. Preventable risk factors for nasal cancer. Ann Epidemiol. 2000;10:186-191.

2. Weymuller EA, Gal T.J. Neoplasms of the nasal cavity. In: Cummings CW, Flint PW, Harker LA et al. (eds.). Otolaryngology - Head and Neck Surgery. 4th. Elsevier Mosby. Pennsylvania. 2005;1212.

3. Zimmer LA Carrau RL. Neoplasms of the nose and paranasal sinuses. In: Bailey BJ, Johnson JT, Newland SD, eds. Head \& Neck Surgery - Otolaryngology. 4th. Lippincott, Williams \& Wilkins. Philadelphia. 2006;417:1480.

4. D'Errico A, Pasian S, Baratti A et al. A case-controlled study on occupational risk factors for sino-nasal cancer. Occup Environ Med. 2009;66:448-455.

5. Barnes L. Schneiderian papillomas and nonsalivary glandular neoplasms of the head and neck. Mod Pathol. 2002;15: 279-297.

6. Von Buchwald C, Bradley PJ. Risks of malignancy in inverted papilloma of the nose and paranasal sinuses. Curr Opin Otolaryngol Head Neck Surg. 2007;15:95-98.

7. Barak Y, Juven T, Haffner R et al. Mdm2 expression is induced by wild type p53 activity. Eur Mol Biol Organ J. 1993;12:461-468.

8. Miyashita T, Krajewski S, Krajewski M et al. Tumor suppressor p53 is a regulator of bcl- 2 and bax gene expression in vitro and in vivo. Oncogene. 1994;9:1799-1805.

9. Deveraux QL, Reed JC. IPA family proteins: suppressors of apoptosis. Genes Dev. 1999;13:239-252.

10. Adida C, Crotty PL, McGrath J et al. Developmentally regulated expression of the novel cancer anti-apoptosis gene survivin in human and mouse differentiation. Am J Pathol. 1998;152:43-49.

11. Fukuda S, Pelus LM. Regulation of the inhibitor-of-apoptosis family member survivin in normal cord blood and bone marrow CD34(+) cells by hematopoietic growth factors: implication of survivin expression in normal hematopoiesis. Blood. 2001;98:2091-2100.

12. Sah NK, Khan Z, Khan GJ et al. Structural, functional and therapeutic biology of survivin. Cancer Lett. 2006;244: 164-171.

13. Swana HS, Grossman D, Anthony JN et al. Tumor content of the antiapoptosis molecule survivin and recurrence of bladder cancer. Lancet. 1999;341:452-453.

14. Yu J, Leung WK, Ebert MPA et al. Increased expression of survivin in gastric cancer patients and in first degree relatives. Br J Cancer. 2002;87:91-97.

15. Ambrosini G, Adida C, Altieri DC. A novel anti-apoptosis gene, survivin, expressed in cancer and lymphoma. Nat Med. 1997;3:917-921.

16. Tanaka K, Iwamoto S, Gon $\mathrm{G}$ et al. Expression of survivin and its relationship to loss of apoptosis in breast carcinomas. Clin Cancer Res. 2000;6:127-134.

17. Izava A, Kobayashi D, Nasu S et al. Relevance of c-erbB-2, PLU-1 and survivin mRNA expression to diagnostic assessment of breast cancer. Anticancer Res. 2002;22:2965-2969.

18. Rodríguez JA, Span SW, Ferreira CG et al. CRM1-mediated nuclear export determines the cytoplasmic localization of the antiapoptotic protein Survivin. Exp Cell Res. 2002;275:44-53.

19. Altieri DC. New wirings in the survivin networks. Oncogene. 2008;27:6276-6284.

20. Mita AC, Mita MM, Nawrocki ST et al. Survivin: key regulator of mitosis and apoptosis and novel target for cancer therapeutics. Clin Cancer Res. 2008;14:5000-5005.

21. Tamm I, Wang Y, Sausville E et al. IAP-family protein survivin inhibits caspase activity and apoptosis induced by Fas

\section{References}


(CD95), Bax, caspases, and anticancer drugs. Cancer Res. 1998;58:5315-5320.

22. Uren AG, Wong L, Pakusch $M$ et al. Survivin and the inner centromere protein INCENP show similar cell-cycle localization and gene knockout phenotype. Curr Biol. 2000;10: 1319-1328.

23. Bourhis E, Hymowitz SG, Cochran AG. The mitotic regulator Survivin binds as a monomer to its functional interactor Borealin. J Biol Chem. 2007;282:35018-35023.

24. D'Alessandro N, Poma P, Montalto G. Multifactorial nature of hepatocellular carcinoma drug resistance: could plant polyphenols be helpful? World J Gastroenterol. 2007;13:2037-2043.

25. Fortugno P, Wall NR, Giodini A et al. Survivin exists in immunochemically distinct subcellular pools and is involved in spindle microtubule function. J Cell Sci. 2002;115:575-585.

26. O'Connor DS, Grossman D, Plescia J et al. Regulation of apoptosis at cell division by $\mathrm{p} 34 \mathrm{cdc} 2$ phosphorylation of survivin. Proc Natl Acad Sci USA. 2000;97:13103-13107.

27. Yang D, Welm A, Bishop JM. Cell division and cell survival in the absence of survivin. Proc Natl Acad Sci USA. 2004;101:15100-15105.

28. Zhao J, Tenev T, Martins LM et al. The ubiquitin-proteasome pathway regulates survivin degradation in a cell cycle-dependent manner. J Cell Sci. 2000;113:4363-4371.

29. Barnes L, Everson JW, Reichart P et al. World Health Organization Classification of Tumours. Pathology and Genetics Head and Neck Tumours. IARC Press Lyon. 2005;15-17:28-32.

30. Li F, Yang J, Ramnath N et al. Nuclear or cytoplasmic expression of survivin: what is the significance? Int $J$ Cancer. 2005;114:509-512.

31. Liang J, Gao S, Zhang $\mathrm{J}$ et al. Expression of Survivin and Bcl-2 in sinonasal inverted papilloma. Lin Chung Er Bi Yan Hou Tou Jing Wai Ke Za Zhi. 2009;23:933-935.

32. Lo Muzio L, Pannone G, Leonardi R et al. Survivin, a potential early predictor of tumor progression in the oral mucosa. J Dent Res. 2003;82:923-928.

33. Grabowski P, Kühnel T, Mühr-Wilkenshoff F et al. Prognostic value of nuclear survivin expression in oesophageal squamous cell carcinoma. Br J Cancer. 2003;88:115-119.

34. Zhang Y, Huang D, Yu G. Survivin expression and its relationship with apoptosis and prognosis in nasal and paranasal sinus carcinomas. Acta Otolaryngol. 2005;125:1345-1350.

35. Wang Y, Kong W, Xiong X et al. The expressions of survivin and VEGF in squamous cell carcinoma of larynx and the correlation between the two marks. Lin Chuang Er Bi Yan Hou Ke Za Zhi. 2005;19:838-841.
36. Yin W, Chen N, Zhang Y et al. Survivin nuclear labeling index: a superior biomarker in superficial urothelial carcinoma of human urinary bladder. Mod Pathol. 2006;19:1487-1497.

37. Ko YH, Roh SY, Won HS et al. Prognostic significance of nuclear survivin expression in resected adenoid cystic carcinoma of the head and neck. Head Neck Oncol. 2010;30:30.

38. Marioni G, Agostini M, Bedin C et al. Survivin and laryngeal carcinoma prognosis: nuclear localization and expression of splice variants. Histopathology. 2012;61:247-256.

39. Katori H, Nozawa A, Tsukuda M. Cell proliferation, apoptosis, and apoptosis inhibition in malignant transformation of sinonasal inverted papilloma. Acta Otolaryngol. 2007;127:540-546.

40. Fan GK, Imanaka M, Yang B et al. Characteristics of nasal inverted papilloma and its malignant transformation: a study of cell proliferation and programmed cell death. Am J Rhinol. 2006;20:360-363.

41. Zheng YF, Chen ZL, Chen HB et al. Expression of survivin, $\mathrm{Bcl}-2$ and p53 during 4-nitro-quinoline 1-oxide-induced rat tongue carcinogenesis. Zhonghua Kou Qiang Yi Xue Za Zhi. 2009;44:168-171.

42. Sun M, Qin Y. Expression of survivin and its correlation with p53 and bcl-2 expression in laryngeal and hypopharyngeal cancer. Lin Chung Er Bi Yan Hou Tou Jing Wai Ke Za Zhi. 2008;22:346-348,351.

43. Bura M, Seiwerth S, Vladika I et al. Possible prognostic significance of p53 and Ki 67 in inverted sinonasal papilloma. Coll Antropol. 2007;31:545-549.

44. Mumbuc S, Karakok M, Baglam T et al. Immunohistochemical analysis of PCNA, Ki67 and p53 in nasal polyposis and sinonasal inverted papillomas. J Int Med Res. 2007;35: 237-241.

45. Gunia S, Liebe D, Koch S. Loss of basal cell keratin 14 reflects increased risk of recurrence in surgically resected sinonasal inverted papilloma. J Clin Pathol. 2008;61:707-712.

46. Hadar T, Shvero J, Yaniv E et al. Human topoisomerase II-alpha is highly expressed in sinonasal-inverted papilloma, but not in inflammatory polyp. J Cell Mol Med. 2008;12: 1551-1558.

47. Kawasaki $\mathrm{H}$, Toyoda $\mathrm{M}$, Shinohara $\mathrm{H}$ et al. Expression of survivin correlates with apoptosis, proliferation, and angiogenesis during human colorectal tumorigenesis. Cancer. 2001;91:2026-2032.

48. Li F, Ambrosini G, Chu EY et al. Control of apoptosis and mitotic spindle checkpoint by survivin. Nature. 1997;396: $580-584$. 